DOI: $10.15593 / 2224-9982 / 2018.53 .08$

УДК 621.77: 629.7.036.34

\author{
И.М. Александров, К.Е. Миляев, С.В. Семёнов
}

Пермский национальный исследовательский политехнический университет, Пермь, Россия

\author{
АНАЛИЗ ВОЗМОЖНОСТИ ПРИМЕНЕНИЯ НИЗКОПЛАСТИЧНОГО \\ ВЫГЛАЖИВАНИЯ ДЛЯ ПОВЫШЕНИЯ НАДЕЖНОСТИ ЛОПАТОК ГТД
}

\begin{abstract}
Повышение поверхностной прочности, износостойкости и коррозионной стойкости является важным направлением в машиностроении. Для получения материалов с нужными характеристиками постоянно разрабатываются новые методы. Их развитие ограничивают такие факторы, как стоимость обработки изделия, трудоёмкость процесса, необходимость в дополнительном оборудовании высокой сложности. Основной задачей в машиностроении является поиск наиболее экономически эффективных методов повышения характеристик обрабатываемых материалов. Рассмотрен метод повышения качества поверхности при финишной холодной обработке под названием «низкопластичное выглаживание» (англ. Low plasticity burnishing). Рассмотрен принцип работы метода, инструмент и станки для выполнения работ. Представлено качественное сравнение с другими методами финишной обработки аналогичного действия, такими как дробеструйная и обработка точением. На основании сравнения и результатов практических испытаний были получены выводы о преимуществах данного метода. Метод низкопластичного выглаживания показал превосходство по производительности над дробеструйной обработкой по результатам многоциклового воздействия на усталость сплава IN718. При сравнении чистового точения с низкопластическим выглаживанием было получено при определенных режимах выглаживания уменьшение шероховатости поверхности в 12,4 раза. Рассмотрен пример применения метода для повышения качества механических свойств вентиляторной лопатки двигателя F404, а также процесс испытаний лопаток, прошедших обработку этим методом, в сравнении с необработанными лопатками. Результаты данных испытаний показали влияние метода низкопластичного выглаживания на повышение прочности лопаток и их сопротивление повреждениям посторонними предметами.

Ключевые слова: пластическое выглаживание, упрочнение лопаток ГТД, усталостная прочность, остаточные напряжения, сопротивляемость повреждениям посторонними предметами.
\end{abstract}

\author{
I.M. Aleksandrov, K.E. Milyaev, S.V. Semenov \\ Perm National Research Polytechnic University, Perm, Russian Federation
}

\title{
APPLICATION OF TECHNOLOGY OF LOW PLASTICITY BURNISHING IN HEIGHTENING OF REABILITY OF FAN BLADES POSSIBILITY ANALYSIS
}

\begin{abstract}
The increase in surface strength, wear resistance and corrosion resistance is an important direction in engineering. To obtain materials with the necessary characteristics, new methods are constantly being developed. Their development is limited by such factors as the cost of processing the product, the laboriousness of the process, the need for additional equipment of high complexity. The main task in engineering is to find the most cost-effective methods for improving the characteristics of processed materials. The method of increasing the surface quality at the finish cold treatment, called low plasticity burnishing, is considered. The principle of work of a method, the tool and machine tools for performance of works is considered. A qualitative comparison with other finishing methods of a similar action is presented, such as shot blasting and turning treatment. Based on the comparison and the results of practical tests, conclusions were drawn about the advantages of this method. Low plasticity burnishing method showed superiority in performance of shot peening on the results of multicycle fatigue effects on the alloy IN718. When comparing fine finishing with low-plastic smoothing, the surface roughness was reduced by 12.4 times under certain smoothing regimes. An example of the application of the method for improving the mechanical properties of the fan blades of the F404 engine is considered, as well as the testing of blades treated with this method with untreated vanes. The results of these tests showed the influence of the low-plastic burnishing method on increasing the strength of the blades and their resistance to damage by foreign objects.

Keywords: low plasticity burnishing, hardening of gas turbine blades, fatigue strength, residual stresses, resistance to damage by foreign objects
\end{abstract}

В настоящее время конструкции авиационных двигателей становятся всё более сложными. В стремлении удовлетворить постоянно растущие требования к характеристикам двигателя конструкторы применяют передовые технологии машиностроения, используют новые материалы и методы конструирования. Отдельного внимания требуют проблемы повышения надежно- 
сти и безопасности двигателя, неразрывно связанные с решением таких задач, как трещиностойкость, прочность и циклическая долговечность его деталей.

Основной причиной поломки рабочих и сопловых лопаток являются усталостные разрушения [1]. Трещина может привести к поломке целой детали, что, в свою очередь, может вызвать серьезные последствия. Одна из основных причин появления трещин и концетраторов напряжений - попадание посторонних предметов в воздухозаборник двигателя $[2,3]$.

Статистический анализ показывает, что количество двигателей, снятых с эксплуатации по причине повреждения посторонними предметами, такими как птицы, частички взлётного покрытия и оставленный на взлётно-посадочной полосе мусор, составляет 50 \% от общего числа досрочно снимаемых двигателей. Так, например, основываясь на данных анализа, правительство США в 1994 г. приняло решение о создании многолетней программы, целью которой является повышение прочности деталей ГДТ. Одной из основных задач стало увеличение допустимых размеров повреждений на кромках лопаток в 15 раз (с 0,127 до 1,875 мм) [4].

Начиная с 2004 г. повышенное внимание уделяется новым методам упрочнения деталей, один из них проанализирован в данной статье [4].

Метод низкопластичного выглаживания (HB) представляет собой многократное выглаживание индентором поверхности с целью сглаживания микрорельефа и пластического деформирования обрабатываемого материала. Он является запатентованным и испытанным методом, относящимся к области машиностроения, и используется для финишной обработки поверхностей [5]. Данная методика совершенствования поверхности металлических деталей была впервые применена в 1996 г. в лаборатории Lambda Technologies и промышленно применяется с 2004 г. [6]. Она позволяет получить глубокие стабильные остаточные напряжения сжатия с меньшим уровнем холодной обработки, чем при традиционном глубоком прокате. Применяется для повышения прочности поверхностного слоя, устойчивости к повреждениям и улучшения многоцикловой усталости, уменьшения уровня холодной обработки и плотности дислокаций вместе с улучшенным удержанием дислокаций полезного сжатия при повышенных температурах. Улучшает сопротивляемость многоцикловой усталости, повышает сопротивляемость повреждениям, коррозийной усталости, коррозионному растрескиванию под напряжением, а также усталостному напряжению при изгибе в компонентах турбин авиационных двигателей, деталей самолётов, подверженных старению, в контейнерах для отработанных ядерных отходов, биомедицинских имплантатах и сварных соединениях [7].

В данный момент метод нашёл применение в конечной обработке шеек ротора турбокомпрессора, дорожек роликоподшипников, поверхностном упрочнении лопаток турбин [8]. Также применяется он в медицине, улучшая свойства металлических протезов, что позволяет избавиться от болезненной и дорогостоящей процедуры замены износившихся частей [9]. Эти примеры не единичные. Благодаря своим выдающимся качествам метод НВ имеет весьма хорошие перспективы во многих областях промышленности.

Преимуществами данного метода являются сравнительно высокая производительность (120-200 м/мин), лёгкость применения на станках с числовым программным управлением, что увеличивает экономическую эффективность и устраняет необходимость содержания отдельного цеха, полностью предназначенного для использования данного метода [10].

Кроме того, НВ уменьшает шероховатость поверхностей, а для таких деталей, как подшипники или зубчатые зацепления, это может стать определяющим при расчёте на тепловыделение [11]. Метод увеличивает прочность конструкции, что достигается за счёт структурирования поверхностного слоя и уменьшения количества дефектов (пор, дислокаций и т.п.), отчего уменьшается концентрация напряжений [12].

В данной статье приведен анализ метода низкопластичного выглаживания индентором, перечислены все его основные достоинства и дана общая информация как об областях его применения, так и об изменениях в структуре материала, прошедшего обработку данным методом. Также статья содержит краткий обзор результатов применения метода НВ в обработке 
входных кромок лопаток газотурбинных двигателей самолёта McDonnel Douglas F/A-18 Hornet [13].

Поверхностная обработка методом низкопластичного выглаживания выполняется с помощью стандартных многоосных станков с ЧПУ [14] для позиционирования обрабатывающего инструмента и гидравлического контроллера, управляющего подачей жидкости, в которой находится выглаживающий шарик (рис. 1).

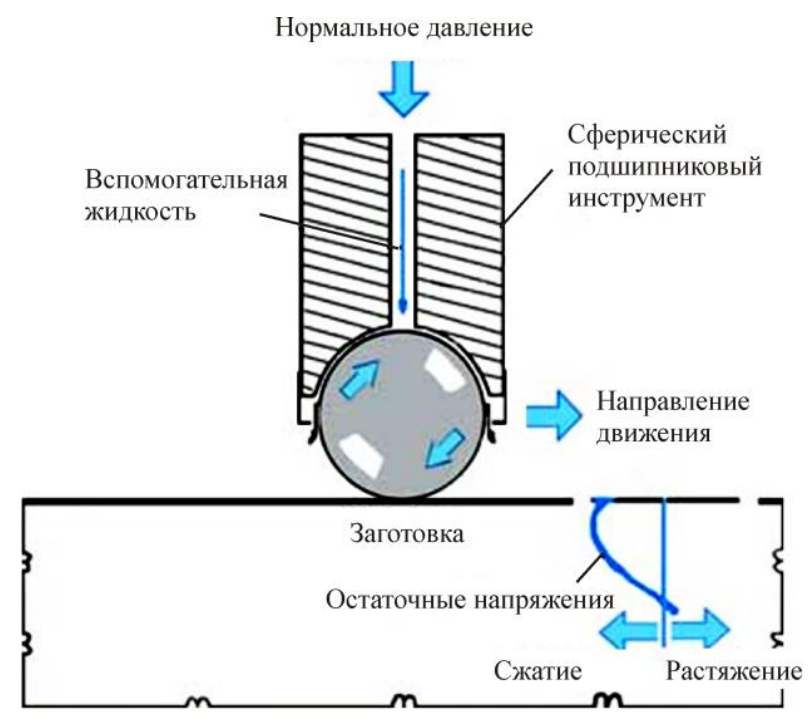

Рис. 1. Принципиальная схема установки [15]

Сила выглаживания, как и положение инструмента, постоянно и одновременно контролируются. Данная процедура позволяет уменьшить количество дефектов на поверхности, не получая при этом наклёпа. Индентор может иметь форму шара. Распространение получили алмазные инденторы и инденторы из мелкодисперсного нитрида бора. Глубина внедрения индентора назначается из соотношения $h=(1,5 \ldots 2) R_{\max }$, где $R_{\max }$ - наибольшая высота профиля поверхности до начала выглаживания [16].

Различают инструменты с жесткой и упругой кинематической связью [17]. Инструменты с жесткой связью позволяют получить точные параметры прикладываемой нагрузки и формы заготовки, однако сильно подвержены автоколебаниям в радиальном направлении, возникающем при движении вдоль обрабатываемой поверхности и при работе инструмента. Инструменты с упругой связью позволяют уменьшать колебания, но при этом передаваемая нагрузка становится меньше, как и производительность. Упругий элемент имеет малый ресурс, поэтому нуждается в частой замене, что накладывает экономические ограничения.

Также предложен способ жесткоупругого исполнения (рис. 2) [18]. Конструкция состоит из следующих элементов: два индентора 1,2 , два винта 3,4 , державка 5 , пружина 6 , винт 7 . При помощи винта 3 , индентор 1 закрепляется в державке 5, а индентор 2 фиксируется от выпадения из державки при помощи винта 4. В державку вставляется пружина 6. Ввинчивая винт 7 в державку 5, осуществляется сжатие пружины 6 на требуемую силу отделочно-упрочняющей обработки. Таким образом, для индентора 1 обеспечивается жесткая кинематическая связь между ним и деталью, а для индентора 2 обеспечивается упругая кинематическая связь между ним и деталью. Смещение применяется для уменьшения высотных параметров шероховатости поверхности, так как второй индентор гарантированно проходит по выступу профиля шероховатости поверхности, образованной первым индентором. 


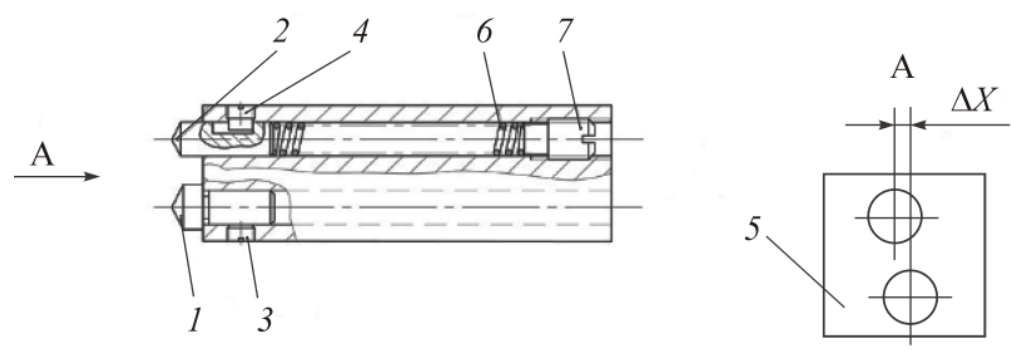

Рис. 2. Жёсткоупругий выглаживатель

Такая конструкция инструмента позволяет комплексно обеспечить параметры качества поверхностного слоя рабочих поверхностей ответственных деталей за счет разных радиусов инденторов: первый индентор необходимо взять меньшего радиуса, чем второй индентор.

Таким образом, первым индентором будут обеспечены максимальные значения микротвердости поверхностного слоя и остаточных напряжений сжатия, а вторым индентором - минимальные значения высотных параметров шероховатости выглаженной поверхности. Для увеличения подачи инструмента и уменьшения износа самого индентора применяется принудительное его вращение, тогда индентор более равномерно изнашивается и нивелируется вероятность его заклинивания $[19,20]$.

Доступно сравнение НВ с дробеструйной обработкой [21]. Дробеструйная (ДС) обработка приводит как к тепловой, так и к механической релаксации. Параметры, задаваемые при методе НВ специальным образом, выбраны так, чтобы создавать глубокий слой с остаточными напряжениями сжатия при объеме холодной обработки, составляющем менее $5 \%$ от нормальной. Остаточные напряжения и процентное количество холодной обработки, профили глубины при обработке ДС-методом и методом НB стали IN718 до и после температурного воздействия в 525 и $600{ }^{\circ} \mathrm{C}$ на протяжении 1000 ч показаны на рис. 3. Остаточные напряжения после дробеструйной обработки на поверхности, где уровень холодной обработки выше всего, почти полностью релаксируются. Как слой, обработанный методом $\mathrm{HB}$, так и более глубокие слои, не подверженные холодной обработке, участки, подвергнувшиеся ДС-обработке, сохраняют количество напряжений. Считается, что термической стабильности приписывается уменьшение плотности дислокаций и, соответственно, ограниченной аннигиляции дислокаций при повышенных температурах, что нивелирует механизм релаксации [22, 23].

Результаты многоциклового воздействия на усталость сплава IN718 (рис. 4) [24] указывают на существенной улучшение производительности метода низкопластичного выглаживания по сравнению с дробеструйной обработкой после воздействия температур как при 525 , так и при $600{ }^{\circ} \mathrm{C}$. Пониженная усталостная прочность при дробеструйной обработке после влияния вышеуказанных температур объясняется потерей поверхностного сжатия (см.
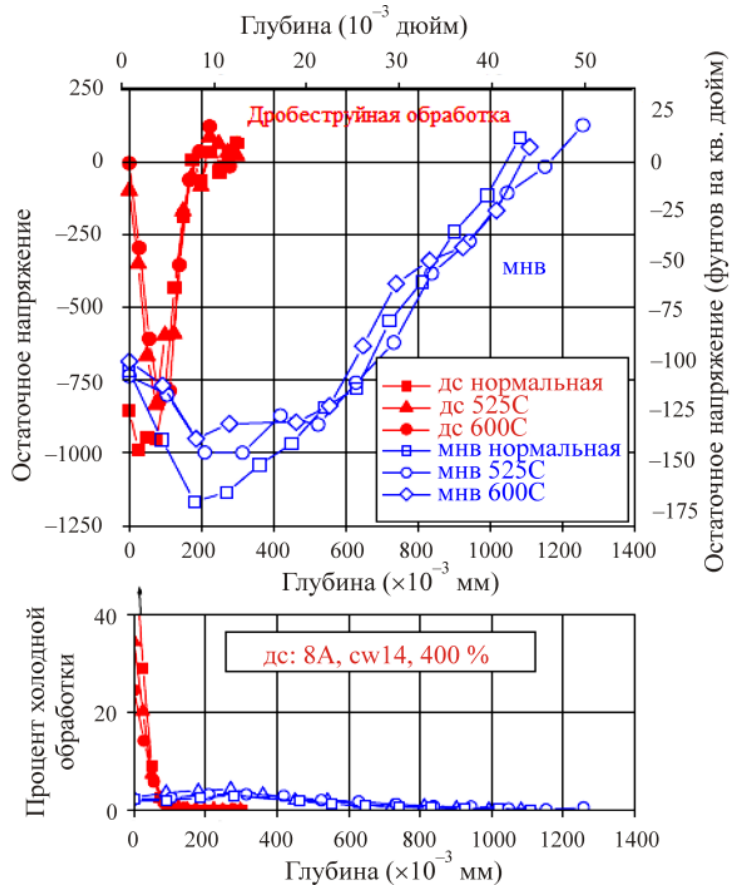

Рис. 3. Сравнение методов ДС и НВ на примере стали IN718

рис 4) [25]. Аналогичные преимущества при усталости с использованием метода НВ описаны на примере обработки сплава Ti-6Al-4V. Метод НB был применён благодаря использованию 
суппорт-инструментов для создания слоя сжатия на гранях тестовых лопаток компрессора сплава Ti-6Al-4V, улучшая устойчивость к напряжениям. При симуляции центробежного среднего напряжения во вращающемся двигателе метод НВ повысил усталостное сопротивление номинально на 207 МПа по сравнению с необработанными лопатками, приблизившись к пределу текучести материала [26,27].

Одним из основных факторов, ведущих к повреждению и разрушению лопаток компрессоров авиационных двигателей, является попадание в них посторонних предметов [28]. Поэтому упрочнение данных элементов является первоочередной задачей, для решения которой может быть использован метод низкопластичного выглаживания.

В большинстве случаев [29] обработанные методом НВ лопатки, испытанные без повреждений посторонними предметами, на таких высоких уровнях воздействия сломались в зонах, где уровень обработки НВ был мал, чаще всего в соединениях типа «ласточкин хвост». Воздействие от постороннего предмета глубиной примерно в 0,5 мм на лопатки, не обработанные НВ, уменьшили усталостную прочность с 689 до 241 МПа. Усталостная прочность обработанных НВ лопаток с воздействием глубиной 0,5 мм примерно равна усталостной прочности неповреждённых лопаток, не обработанных НВ [30]. Более глубокое воздействие уменьшило усталостную прочность обработанных лопаток на $10 \%$ в сравнении с необработанными и неповрежденными. Подобные достижения были также получены на лопатках из титанового сплава Ті-6-2-4-2 и нержавеющей стали 17-4РН [31].

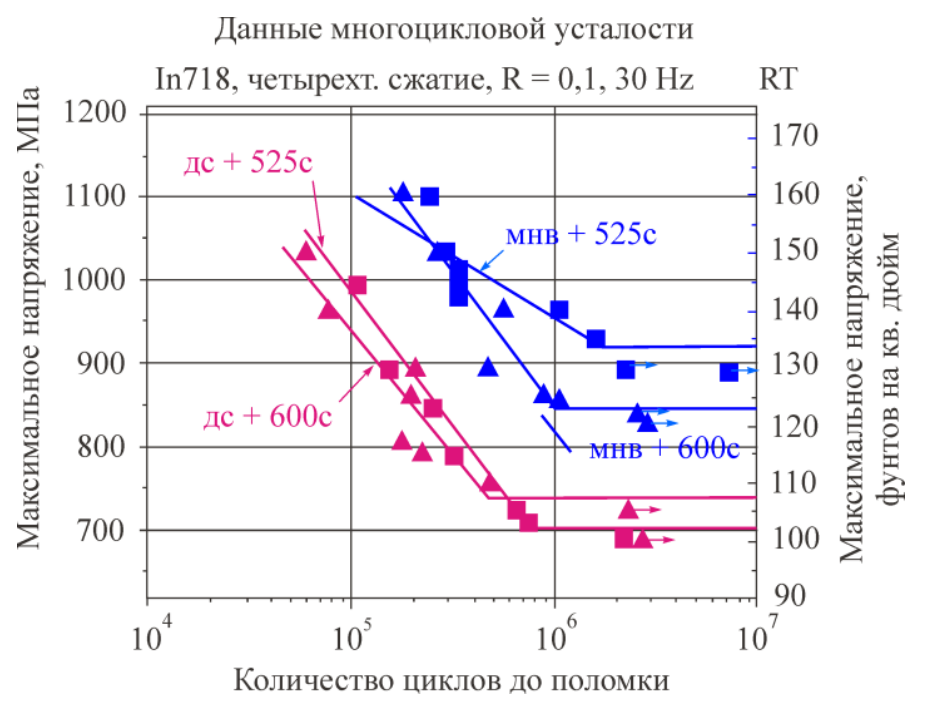

Рис. 4. Воздействие многоцикловой усталости на сплав IN718 [32]

Наглядным примером сравнения является сравнение чистового точения и НВ [33]. Было проведено исследование на образцах из стали 20X13 термообработанной до состояния низкоотпущенного реечного мартенсита. Диски из термоупрочненной стали подвергали чистовому точению и финишной обработке выглаживанием алмазным индентором, имеющим форму шара радиусом 4 мм.

Результаты исследования показали, что при финишной обработке образцов силой $P=230 \mathrm{H}$, что является рекомендуемым для данного типа сталей, происходит не полное сглаживание исходной шероховатости с частичным деформированием вершин неровностей (рис. 5) [33].

Вершины и впадины, обработанные НВ, имеют остаточные следы обработки точением. Увеличение силы выглаживания до $P=340$ Н позволяет полностью сгладить вершины микронеровностей поверхности от предварительного чистового точения (рис. 6) [33]. Результаты исследования приведены в таблице [33]. 

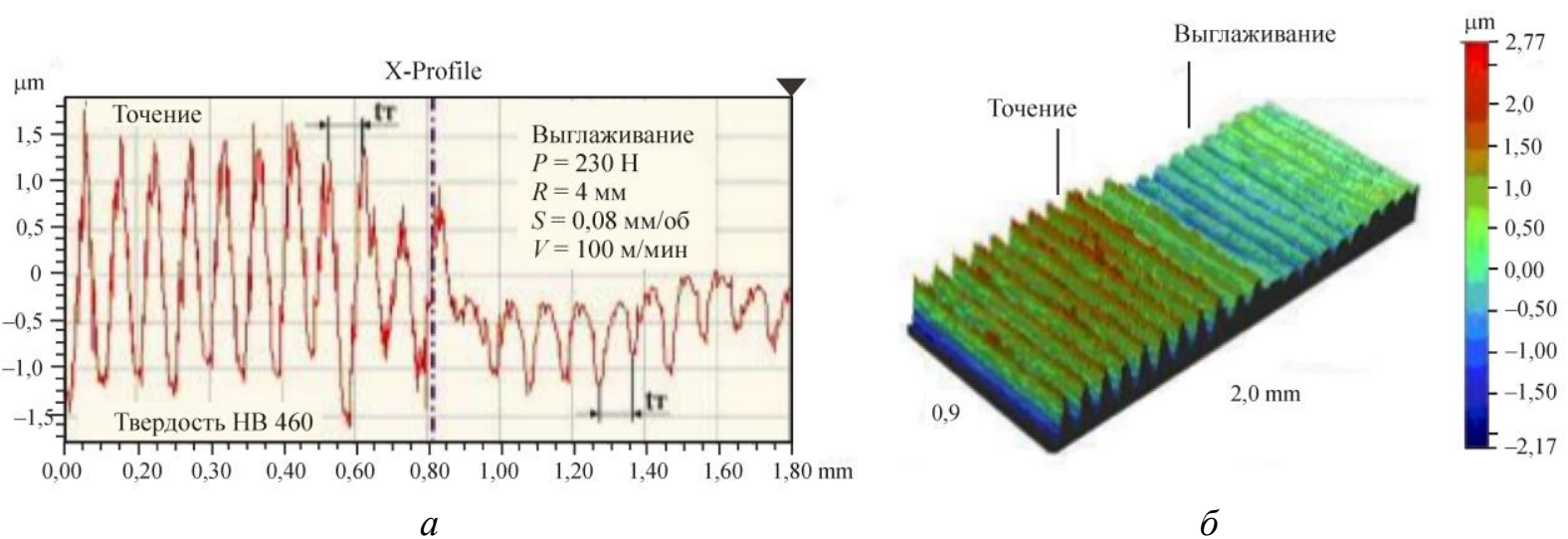

Рис. 5. Профилограмма ( $a$ ) и трехмерное изображение (б) на оптическом профилометре при воздействии с силой $P=230 \mathrm{H}$
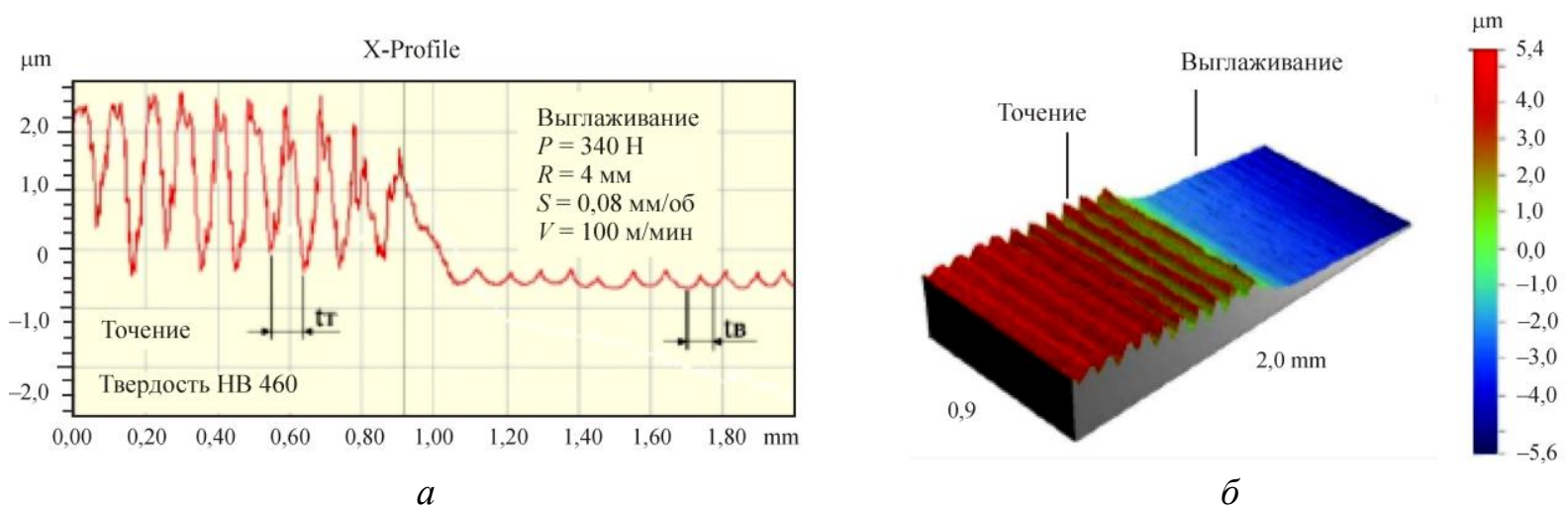

Рис. 6. Профилограмма ( $a$ ) и трехмерное изображение (б) на оптическом профилометре при воздействии с силой $P=340 \mathrm{H}$

Шероховатость поверхности образцов 20Х13 после чистового точения и выглаживания [33]

\begin{tabular}{|c|c|c|}
\hline \multirow{2}{*}{ Сила выглаживания, $\mathrm{H}$} & \multicolumn{2}{|c|}{ Параметр шероховатости Rа, мкм } \\
\cline { 2 - 3 } & Чистовое точение & Выглаживание \\
\hline 230 & 0,80 & 0,24 \\
\hline 340 & 1,12 & 0,09 \\
\hline
\end{tabular}

Результаты, представленные на рис. 5 и 6, свидетельствуют о том, что основополагающим фактором при выглаживании является сила давления на индентор. При рекомендованном давлении $(P=230 \mathrm{H})$ процесс выглаживания давал уменьшение шероховатости в 3,3 раза. При увеличении силы давления до $P=340 \mathrm{H}$ наблюдалось значительное уменьшение параметров шероховатости до 12,4 раза.

Удачным примером практического применения метода низкопластичного выглаживания является его применение для создания слоя с высокой величиной остаточных напряжений на ведущей кромке вентиляторной лопатки двигателя F404 самолета F18 [3]. Целью было улучшение устойчивости к повреждениям диаметром от 0,13 до 0,5 мм. Была получена зона сжатия по толщине порядка 690 МПа. Выполнено тестирование консольной усталости при $R=0,1$, проведено тестирование на повреждения инородными предметами с использованием 60 -градусной вырезки. Результаты обработки показали устойчивость к повреждениям инородными предметами до номинального значения в 1,3 мм по глубине, что создаёт слой остаточного напряжения 
сжатия в титановом сплаве Ti-6Al-4V, приближаясь к пределу текучести сплава. Использование метода низкопластичного выглаживания показало улучшение сопротивления к усталостным нагрузкам и устойчивости к повреждениям (на порядок) в стальных, титановых, никелевых и алюминиевых сплавах.

Для проведения исследований были взяты десять лопаток с дефектами, накопленными за время эксплуатации двигателя. Искусственно на входной кромке части лопаток имитировались повреждения в виде разрезов различной глубины или следов от высокоскоростного столкновения со стальными шарами. Затем лопатки с искусственной и эксплуатационной деформацией подвергли низкопластичному выглаживанию. Выглаживание осуществлялось сферическим роликом под давлением, достаточным для деформации поверхности материала, создавая, таким образом, сжатый слой с высокими остаточными напряжениями. Низкий уровень холодной обработки позволил обеспечить стабильность остаточного сжатия. Управление инструментом осуществлялось на станке с ЧПУ (рис. 7).

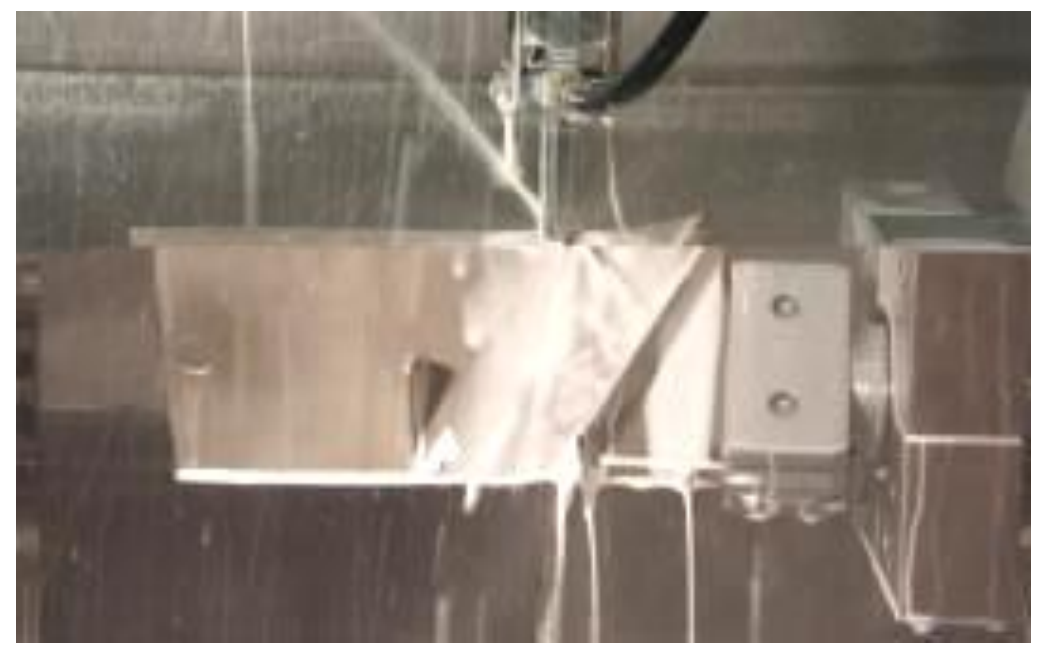

Рис. 7. Обработка лопатки первой ступени двигателя F404 [3]

Параметры обработки были определены с помощью серии экспериментов путем варьирования давления инструмента и его траектории таким образом, чтобы были достигнуты необходимые уровни поверхностных и глубинных напряжений.

\section{Заключение}

Из анализа публикаций можно сделать вывод, что в результате многочисленных экспериментов, проведённых на различных сплавах, подвергнутых различным температурным, физическими и химическим воздействиям, продемонстрировано, что метод НВ обладает преимуществом над дробеструйной обработкой материалов. Применение данного метода позволяет серьезно улучшить физические свойства материала, что в перспективе даёт возможность серьёзного упрощения процесса обработки металлических деталей, а также удешевления данного процесса.

Технологически данный процесс представляет собой более развитое применение методов обкатки роликом или шариком, давно устоявшихся в производстве. Основным отличием является то, что прижатие шарика к поверхности осуществляется струёй жидкости под регулируемым давлением. Усилие прижатия шарика существенно меньше, чем в других методах, а количество проходов по одному и тому же месту увеличивается.

Глубокий уровень сжатия позволяет ослабить влияние повреждений от посторонних предметов глубиной до 0,25 мм в сплавах Ti-6Al-4V, IN718 и 17-4PН на прочностные свойства 
лопаток. Коррозионная усталостная прочность сплава 17-4РН после обработки НВ и износостойкость увеличились, что повысило износостойкость сплава, увеличив срок работы лопаток. На данный момент ведутся исследования результатов обработки других сплавов, показывая сходные результаты.

Так, было показано, что НВ может быть проведён на обычных станках ЧПУ в помещении заводского цеха или при проведении техосмотра и ремонта, существенно упрощая его применение. Применение подобного метода в производстве не требует значительных финансовых затрат, и может производиться, как уже было сказано ранее, на обычных фрезерных станках. Однако для функционирования данного метода необходимо использование заранее заданных программ обработки, которые создаются на основе упрочнения и испытаний специальных образцов, имитирующих кромки деталей.

Из этого следует, что процессу обработки методом НВ можно подвергать не только недавно произведённые детали, но и те, что проходят процесс ремонта, это позволит увеличить срок их службы.

Все это говорит о больших перспективах метода низкопластичного выглаживания для финишной обработки лопаток и других узлов газотурбинных двигателей.

\section{Список литературы}

1. Лукьянов А.А., Бобровский И.Н., СевостьяновА.С. Обзор режимов обработки металлов поверхностно-пластическим деформированием // Инновации в науке. - 2014. - С. $23-28$.

2. Нихамкин М.Ш., Воронов Л.В., Семенова И.В. Концентрация напряжений в лопатках компрессора при повреждении их посторонними предметами // Изв. вузов. Авиационная техника. - 2011. - № 4. C. $15-18$.

3. Nikhamkin M.S., Voronov L.V., Semenova I.V. Effect of blade geometry and foreign object kinetic energy on blades damage. Proceedings of the ASME Turbo Expo Cep. ASME Turbo Expo 2010: Power for Land, Sea, and Air, GT 2010. - 2010. - P. 505-510.

4. Разработка и применение новых методов упрочнения деталей ГДТ, основанных на пластическом деформировании поверхностных слоёв (Обзор) / Ю.А. Ножницкий, А.В. Фишгойт, Р.И. Ткаченко, С.В. Теплова // Общие вопросы двигателестроения. - 2006. - № 2. - С. 4-7.

5. Prevey P.S., Hombach D.J., Jacobs T.L. Improved Damage Tolerance in Titanium Alloy Fan Blades with Low Plasticity Burnishing // Lambda Research. - 2002.

6. Суслов А.Г., Гуров Р.В., Тишевских Е.С. Отделочно-упрочняющая обработка поверхностным пластическим деформированием // Упрочняющие технологии и покрытия. - 2008. - № 9. - С. 20-21.

7. Stress Concentration: Lambda Technologies Group. - 2014. URL: http://www.lambdatechs.com/ stress-concentration.html.

8. Tanuma K., Man C.S., Chen Y. Dispersion of Rayleigh waves in weakly anisotropic media with vertically inhomogeneous initial stress // International Journal of Engineering Science. - 2015. - P. 63-82. DOI: $10.1016 /$ j.ijengsci.2015.03.001

9. Афонин А.Н., ЛаринА.И., Макаров А.В. Гетерогенное упрочнение деталей горно-металлургических машин поверхностным пластическим деформированием // Изв. вузов. Черная металлургия. 2015. - C. 823-827. DOI: 10.17073/0368-0797-2015-11-823-827

10. Uddin M.S., Hall C., Murphy P. Surface treatments for controlling corrosion rate of biodegradable MG and MG based alloy implants // Science and Technology of Advanced Materials. - 2015. DOI: $10.1088 / 1468-6996 / 16 / 5 / 053501$

11. Toward control of subsurface strain accumulation in nanostructuring burnishing on thermostrengthened steel / V.P. Kuznetsov, I. Yu. Smolin, A.I. Dmitriev [et al.] // Surface and Coatings Technology. - 2016. P. 171-178. DOI: 10.1016/j.surfcoat.2015.11.045

12. Low Plasticity Burnishing: Lambda Technologies Group. - 2010. - URL: http://www.lambdatechs.com/ low-plasticity-burnishing-LPB.html.

13. Одинцов Л.Г. Финишная обработка деталей алмазным вибровыглаживанием. - М.: Машиностроение, 1981. $-160 \mathrm{c}$.

14. The Influence of Surface Enhancement by Low Plasticity Burnishing on the Corrosion Fatigue Performance of AA7075-T6. - 2014. - URL: http://www.lambdatechs.com/documents/230.pdf\#search=\%22LPB\% 22 . 
15. Способ упрочняющей обработки поверхностей деталей выглаживанием [Электронный ресурс] / P.А. Сааврай, В.П. Кузнецов, А.Е. Киряков [и др.]. - URL: http://www.findpatent.ru/patent/245/2458777.html.

16. Кузнецов В.П. Способ наноструктурирующего упрочнения поверхностного слоя прецизионных деталей выглаживанием [Электронный ресурс]. - URL: http://www.freepatent.ru/patents/2460628.

17. Gulhane U.D., Mishra S.B., Mishra P.K. Enhancement of surface roughness of 3161 Stainless steel and ti-6al-4v using low plasticity Burnishing: doe approach // Research Scholar, Department of Mechanical Engineering. Motilal Nehru National Institute of Technology, Allahabad (U.P.). - 2004.

18. Influence of ultrasonic shot peening on corrosion behavior of 7075 aluminum alloy / V. Pandey, K. Chattopadhyay, N.C.S. Srinivas [et al.] // Journal of Alloys and Compounds. - 2017. - P. 826-840. DOI: 10.1016/j.jallcom.2017.06.310

19. Губанов В.Ф. Выглаживание: качество, технологии и инструменты. - М.: Изд-во Акад. естествознания, 2013.

20. Experimental study and finite element analysis based on equivalent load method for laser ultrasonic measurement of elastic constants / Y. Zhan, Z. Qiu, C. Liu, F. Zhang // Ultrasonics. - 2016. - P. $243-247$. DOI: 10.1016/j.ultras.2016.03.014

21. Tang J., Luo H.Y., Zhang Y.B. Enhancing the surface integrity and corrosion resistance of Ti-6Al-4V titanium alloy through cryogenic burnishing // International Journal of Advanced Manufacturing Technology. 2017. - P. 2785-2793. DOI: 10.1007/s00170-016-9000-y

22. Gao Y. Influence of different surface modification treatments on surface integrity and fatigue performance of TC4 titanium alloy // Jinshu Xuebao. - 2016. - P. 915-922. DOI: 10.11900/0412.1961.2015.00628

23. John T. Cammett, Paul S. Prevey "Fatigue strength restoration in corrosion pitted 4340 alloy steel via low plasticity burnishing" // Lambda Research, Cincinnati, OH. - 2013. - URL: https://www.lambdatechs.com/wp-content/uploads/228.pdf.

24. Prevéy P. The Effect of Cold Work on the Thermal Stability of Residual Compression in Surface Enhanced IN718 / Proc. 20th ASMI Conference. - 2000.

25. Dynamic Fracture in Carbon-fibre Composites: Effect of Steel and Ice Projectiles / L.A. Coles, A. Roy, L. Voronov, S. Semyonov, M. Nikhamkin, V. V. Silberschmidt // Procedia Structural Integrity. - 2016. Vol. 2. - C. 366-372. DOI: 10.1016/j.prostr.2016.06.047., Web of Science.

26. Prevéy P. The effect of cold work on the thermal stability of residual compression in surface enhanced IN718. - Lambda Research, Cincinnati, OH. - 2000.

27. Prevéy P. Application of low plasticity burnishing to improve damage tolerance of a ti-6al-4v first stage fan blade // Proc. 20th ASMI Conference. - 2000.

28. Damage Tolerance Improvement of Ti-6-4 Fan Blades with Low Plasticity Burnishing. - 2014. URL: http://www.lambdatechs.com/documents/231.pdf\#search.

29. Improved Damage Tolerance Of Ti-6AL-4V Aero Engine Blades And Vanes Using Residual Compression By Design. - 2010. - URL: http://www.lambdatechs.com/documents/262.pdf\#search=\%22LPB\%22.

30. Cammett J.C., Prevéy P.S. Fatigue Strength Restoration in Corrosion Pitted 4340 Alloy Steel via Low Plasticity Burnishing (2001). - 2002. - URL: http://scholar.google.com.

31. Зиновьев Д.В., Уваров Л.Б. Повышение эффективности технологических процессов фрезерования проточной части лопаток компрессора ГТД с приложением вдоль пера растягиваюеей силы // Технология и оборудование механической и физико-технической обработки. - 2009. - № 4. - С. 41-46.

32. Финишная обработка термоупрочняемой высокохромистой стали однопроходным алмазным выглаживанием на токарно-фрезерном центре инструментом с узлом динамической стабилизации / В.П. Кузнецов, А.В. Макаров, Р.А. Саврай [и др.] // Вестник научно-технического развития. - 2011.

\section{References}

1. Lukyanov A.A., Bobrovskiy I.N., Sevostyanov A.S. Obzor rezhimov obrabotki metallov poverkhnostno-plasticheskim deformirovaniyem [An overview of modes of processing of metals by surface plastic deformation]. Innovatsii v nauke, Novosibirsk, 2014, pp. 23-28.

2. Nihamkin M. Sh., Voronov L. V., Semenova I. V. Kontsentratsiya napryazheniy v lopatkakh kompressora pri povrezhdenii ikh postoronnimi predmetami [Stress concentration in compressor blades at their damage by foreign objects]. Izvestiya vysshikh uchebnykh zavedenii. Russian Aeronautics, 2011, no. 4, pp. 15-18.

3. Nikhamkin M.S., Voronov L.V., Semenova I.V. Effect Of Blade Geometry And Foreign Object Kinetic Energy On Blades Damage. Proceedings of the ASME Turbo Expo Cep. "ASME Turbo Expo 2010: Power for Land, Sea, and Air, GT 2010", 2010, P. 505-510. 
4. Nozhnitskiy, U.A, Fishgoit A.V, Tkachenko R.I, Teplova S.V. Razrabotka i primeneniye novykh metodov uprochneniya detaley GDT, osnovannykh na plasticheskom deformirovanii poverkhnostnykh sloyev (Obzor) [Development and application of new methods of hardening of parts of GDE based on plastic deformation of surface layers (Review)]. Obshchiye voprosy dvigatelestroyeniya, 2006, no. 2 (2006), pp. 4-7.

5. Prevey P.S., Hombach D.J., Jacobs T.L. Improved Damage Tolerance in Titanium Alloy Fan Blades with Low Plasticity Burning. Lambda Research, 2002.

6. Suslov A.G., Gurov R.V., Tishevskikh E.S. Otdelochno-uprochnyayushchaya obrabotka poverkhnostnym plasticheskim deformirovaniyem [Finishing-hardening treatment of surface plastic deformation]. $U p$ rochnyayushchiye tekhnologii i pokrytiya, 2008, no. 9, pp. 20-21.

7. Stress Concentration: Lambda Technologies Group [Electronic resource]. 2014. URL: http://www.lambdatechs.com/stress-concentration.html

8. Tanuma K. Dispersion of Rayleigh waves in weakly anisotropic media with verti-cally inhomogenic initial stress / K. Tanuma, C.S. Man, Y. Chen // international journal of engineering science, 2015, pp. 63-82. DOI: 10.1016/j.ijengsci.2015.03.001

9. Afonin A.N., Larin A.I., Makarov A.V. Geterogennoye uprochneniye detaley gorno-metallurgicheskikh mashin poverkhnostnym plasticheskim deformirovaniyem [Heterogeneous hardening of parts of mining and metallurgical machinery by surface plastic deformation]. Izvestiya. Ferrous Metallurgy, 2015, pp. 823-827. DOI: 10.17073/0368-0797-2015-11-823-827

10. Uddin M. S. Surface treatments for controlling corrosion rate of biodegradable MG and MG based alloy implants / M.S. Uddin, C. Hall, P. Murphy // Science and technology of advanced materials, 2015. DOI: 10.1088/1468-6996/16/5/053501

11. Kuznetsov V.P. Howard control of subsurface strain accumulation in nanostructure-ing burning on thermostrengthened steel / V.P. Kuznetsov, I.Yu. Smolin, A.I. Dmitriev [et al.] // Surface and coatings technology, 2016, pp. 171-178. DOI: 10.1016/j.surfcoat.2015.11.045

12. Low Plasticity Burning: Lambda Technologies Group [electronic resource]. 2010. URL: http://www.lambdatechs.com/low-plasticity-burnishing-LPB.html

13. Odintsov L.G. Finishnaya obrabotka detaley almaznym vibrovyglazhivaniyem [Finish machining of parts with a diamond fibromyalgia]. Moscow: Mashinostroyeniye, 1981, 160 p.

14. The Influence of Surface Enhancement by Low Plasticity Burnishing on the corrosion Fatigue Performance of AA7075-T6. [Electronic resource.] 2014. URL: http://www.lambdatechs.com/documents/ 230.pdf\#search=\%22LPB\%22.

15. Savrai R.A., Kuznetsov V.P., Kiryakov A.E. [et al.]. Sposob uprochnyayushchey obrabotki poverkhnostey detaley vyglazhivaniyem [Method of hardening surface treatment of parts by dragging]. Resource URL: http://www.findpatent.ru/patent/245/2458777.html

16. Kuznetsov V.P. Sposob nanostrukturiruyushchego uprochneniya poverkhnostnogo sloya pretsizionnykh detaley vyglazhivaniyem [Method of nanostructuring hardening of the surface layer of precision parts by smoothing]. El. Resource URL: http://www.freepatent.ru/patents/2460628

17. Gulhane U.D., Mishra S.B., Mishra P.K. "Enhancement of surface roughness of 3161 Stainless steel and ti-6al-4v using low plasticity Burnishing: doe approach" research Scholar, Department of Mechanical Engineering, Motilal Nehru National Institute of Technology, Allahabad (U. P.), 2004, India.

18. Pandey V., Chattopadhyay K., Srinivas N.C.S. [et al.]. Influence of ultrasonic shot peening on corrosion behavior of 7075 aluminum alloy. Journal of alloys and compounds, 2017, pp. 826-840. DOI: 10.1016/j.jallcom.2017.06.310

19. Gubanov V.F. Vyglazhivaniye: kachestvo, tekhnologii i instrumenty [Smoothing: quality, technologies and tools]. Akademiya estestvoznaniya, 2013.

20. Zhan Y., Qiu Z., Liu C., Zhang F. Experimental study and finish element analysis based on equivalent load method for laser ultrasonic measurement of elastic constants. Ultrasonics, 2016, pp. 243-247. DOI: 10.1016/j.ultras.2016.03.014

21. Tang J., Luo H.Y., Zhang Y.B. Enhancing the surface integrity and corrosion resistance of Ti-6Al-4V titanium alloy through cryogenic burnishing. The international journal of advanced manufacturing technology, 2017, pp. 2785-2793. DOI: 10.1007/s00170-016-9000-y

22. GAO Y. Influence of different surface modification treatments on surface integrity and fatigue performance of TC4 titanium alloy. Jinshu xuebao, 2016, pp. 915-922. DOI: 10.11900/0412.1961.2015.00628 
23. John T. Cammett, Paul S. Prevey. Fatigue strength restoration in corrosion pitted 4340 alloy steel via low plasticity burning. Lambda Research, Cincinnati, OH, [electronic resource]. 2013. URL: https://www.lambdatechs.com/wp-content/uploads/228.pdf

24. Prevéy P. The Effect of Cold Work on the Thermal Stability of Residual Compression in Surface Enhanced IN718. Proc. 20th ASMI Conference, 2000.

25. Coles L.A., Roy A., Voronov L., Semyonov S., Nikhamkin M., Silberschmidt V.V. Dynamic Fraction in Carbon-fibre Composites: Effect of Steel and Ice Projectiles / // Procedia Structural Integrity [Electronic resource]. 2016, vol. 2, pp. 366-372. DOI: 10.1016/ j.prostr.2016.06.047.

26. Prevéy P. The effect of cold work on the thermal stability of residential compression in surface enhanced in718, Lambda Research, Cincinnati, OH, 2000.

27. Prevéy P. Application of low plasticity burnishing to improve damage tolerance of a ti-6al-4v first stage fan blade, Proc. 20th ASMI Conference, 2000.

28. Damage Tolerance Improvement of Ti-6-4 Fan Blades with Low Plasticity Burnish-ing [electronic resource]. 2014. URL: http://www.lambdatechs.com/documents/231.pdf\#search

29. Improved Damage Tolerance Of Ti-6AL-4V Aero Engine Blades And Vanes Using Residual Compression By Design. El Republic. resource. 2010. URL: http://www.lambdatechs.com/documents/ 262.pdf\#search=\%22LPB $\% 22$.

30. Cammett J. C. and Prevéy P.S. Fatigue Strength Restoration in Corrosion Punched 4340 Alloy Steel via Low Plasticity Burning (2001). 2002. URL http://scholar.google.com.

31. Zinoviev D.V., Uvarov L.B. Povysheniye effektivnosti tekhnologicheskikh protsessov frezerovaniya protochnoy chasti lopatok kompressora GTD s prilozheniyem vdol pera rastyagivayushchey sily [Improving the efficiency of technological processes of milling of the flow part of the compressor blades GTD with accretion along the pen of the tensile force]. Tekhnologiya i oborudovaniye mekhanicheskoy i fiziko-tekhnicheskoy obrabotki, 2009, no. 4, pp. 41-46.

32. Kuznetsov V.P., Makarov A.V., Savray R.A. [et al.]. Finishnaya obrabotka termouprochnyayemoy vysokokhromistoy stali odnoprokhodnym almaznym vyglazhivaniyem na tokarno-frezernom tsentre instrumentom s uzlom dinamicheskoy stabilizatsii [Method of nanostructuring hardening of the surface layer of precision parts by smoothing]. BHTP Vestnik, 2011, 17 p.

\section{Об авторах}

Александров Илья Максимович (Пермь, Россия) - студент кафедры «Авиационные двигатели» ФГБОУ ВО ПНИПУ (614990, г. Пермь, Комсомольский пр., д. 29, e-mail: Ilyaalexa@ yandex.ru).

Миляев Кирилл Евгеньевич (Пермь, Россия) - студент кафедры «Авиационные двигатели» ФГБОУ ВО ПНИПУ (614990, г. Пермь, Комсомольский пр., 29, e-mail: daloros@inbox.ru).

Семенов Сергей Валерьевич (Пермь, Россия) - ассистент кафедры «Авиационные двигатели» ФГБОУ ВО ПНИПУ (614990, г. Пермь, Комсомольский пр., 29, e-mail: sergey.semyonov@ mail.ru).

\section{About the authors}

Ilya M. Aleksandrov (Perm, Russian Federation) - Student, Department of Aircraft engines, Perm National Research Polytechnic University (29, Komsomolsky av., Perm, 614990, Russian Federation, e-mail: Ilyaalexa@yandex.ru).

Kirill E. Milyayev (Perm, Russian Federation) - Student, Department of Aircraft engines, Perm National Research Polytechnic University (29, Komsomolsky av., Perm, 614990, Russian Federation, e-mail: daloros@inbox.ru).

Sergey V. Semenov (Perm, Russian Federation) - Assistant, Department of Aircraft engines, Perm National Research Polytechnic University (29, Komsomolsky av., Perm, 614990, Russian Federation, e-mail: sergey.semyonov@mail.ru).

Получено 18.05.2018 